\title{
O DESAFIO DE IMPLANTAR A SISTEMATIZAÇÃO DA ASSISTÊNCIA DE ENFERMAGEM SOB A ÓTICA DE DISCENTES
}

\author{
The Challenge of to Implant the Nursing Assistance \\ Systematization According to the Students' View \\ El Desafío de Implantar la Sistematización de la \\ Asistencia de Enfermería Según la Óptica de los Discentes
}

\author{
Lucimar Ramos Ribeiro Gonçalves \\ Lídya Tolstenko Nogueira
}

\author{
Inez Sampaio Nery \\ Elisiane Gomes Bonfim
}

\begin{abstract}
Resumo
Estudo com abordagem qualitativa objetivando discutir aspectos relativos ao projeto de implantação da Sistematização da Assistência de Enfermagem - SAE em uma maternidade pública, segundo a ótica de discentes de enfermagem da UFPI. 0 estudo foi desenvolvido com 28 alunos, por meio de relatos escritos, agrupados em três categorias, cuja análise dos conteúdos possibilitou a caracterização das unidades de significação. Os resultados mostraram que a SAE, apesar da resistência inicial dos profissionais de saúde à sua adoção, favoreceu o aprendizado do cuidado de enfermagem e a assistência oferecida à clientela e repercutiu na satisfação discente, das usuárias do serviço e de familiares. Na operacionalização da SAE foram apontadas dificuldades relativas ao preenchimento dos formulários adotados. Os discentes valorizaram a SAE como instrumento metodológico necessário ao desempenho da prática profissional, bem como consideraram que a participação na implantação do projeto tornou oportunas as condições para exercitá-la com autonomia na vida profissional.
\end{abstract}

Palavras-chave: Saúde da Mulher. Enfermagem. Assistência de Enfermagem.

\begin{abstract}
Study with qualitative approach objectifying to argue relative aspects to the project of implantation of the Systematization of the Assistance of Nursing - SAE in a public maternity, according to the view of the nursing students of the UFPI. The study was developed with 28 students, by written stories, grouped in three categories, whose analysis of the contents made possible the characterization of the signification units. The results showed that the SAE, although the initial resistance of the health professionals to its adoption, favored the learning of the nursing care and the assistance offered to the clientele, reflecting in the satisfaction of the students, of the users of the service and of the family. In the operating of the SAE was pointed difficulties related to the fulfilling of the adopted. The students valued the SAE as a necessary methodological instrument to the performance of the professional practice, as well had considered that the participation in the implantation of the project make possible the conditions to exercise it with autonomy in the professional life.
\end{abstract}

\section{Resumen}

Estudio con abordaje cualitativa con objetivo de discutir los aspectos relativos al proyecto de implantación de la Sistematización de la Ayuda de Enfermería - SAE en una maternidad pública, según la óptica de discentes de enfermería de la UFPI. El estudio fue desarrollado con 28 estudiantes, por medio de relatos escritos, agrupados en tres categorías, cuyo análisis del contenido hizo posible la caracterización de las unidades de significación. Los resultados demostraran que la SAE, aunque la resistencia inicial de los profesionales de salud a su adopción, favoreció el aprendizaje del cuidado de enfermería y la ayuda ofrecida a la clientela y repercutió en la satisfacción de los discentes, de las usuarias del servicio y de la familia. En la operacionalización de la SAE fueran apuntadas dificultades relativas a rellenar el formulario adoptado. Los discentes valoraran la SAE como instrumento metodológico necesario al funcionamiento de la práctica profesional, bien como consideraron que la participación en la implantación del proyecto hizo posible las condiciones para ejercitarla con autonomía en la vida profesional.
Keywords:

Women's Health. Nursing. Nursing Care.
Palabras clave:

Salud de la Mujer. Enfermería. Atención de Enfermería. 


\section{INTRODUÇÃO}

A enfermagem tem procurado estabelecer seus objetivos, lidando com aspectos existenciais do impacto da enfermidade sobre os indivíduos e familiares, fazendo disso seu foco de ação. A assistência sistematizada é orientada para as necessidades humanas básicas afetadas pelo processo da doença, visa a auxiliar as pessoas a alcançarem equilíbrio harmônico através da promoção do autoconhecimento, autocura, autocontrole, autorespeito e a terem um entendimento do significado dos acontecimentos que estão fazendo parte de sua vida' ${ }^{1}$

A Sistematização da Assistência de Enfermagem - SAE é reconhecida pelos profissionais de enfermagem como marco a ser institucionalizado nos serviços de saúde. 0 Conselho Federal de Enfermagem, através da Resolução COFEN n $272 /$ 2002, dispõe sobre a SAE nas instituições de saúde no Brasil e determina que sua implementação deva ocorrer em todas as instituições de saúde, tanto públicas quanto privadas. A sistematização deverá ser registrada formalmente no prontuário do cliente e deve conter o histórico de enfermagem, exame físico, diagnóstico, prescrição e evolução de enfermagem .

Entretanto, a despeito do respaldo legal, a prática da SAE não se faz sentir no Piauí com a extensão desejada, tendo em vista serem esparsas e descontínuas as tentativas de adoção desta metodologia assistencial na realidade dos serviços de saúde.

Estudo realizado em Teresina constatou que apesar do Curso de Enfermagem da UFPI, ao longo dos seus 25 anos, ter trabalhado com as teorias e metodologias dos cuidados de enfermagem voltados para as Necessidades Humanas Básicas de "Wanda Horta", a maioria de seus egressos não desenvolve a SAE na prática profissional cotidiana em instituições de saúde públicas e ou privadas em Teresina-PI ${ }^{1}$. Essa realidade mostra a desarticulação entre ensino e prática assistencial e o baixo impacto da academia para a transformação dos serviços de saúde na prestação da assistência.

Assim, na busca de alternativas para conciliar os valores profissionais às necessidades dos discentes de enfermagem $\mathrm{e}$ à realidade dos serviços de saúde, nos quais a prática de enfermagem se apresentava fragmentada e descontínua, comprometendo a qualidade da assistência e do ensino, as autoras propuseram um projeto de extensão denominado "Implantação da Sistematização da Assistência de Enfermagem numa Maternidade Pública em Teresina-Pl".

As inquietações e reflexões coletivas conduziram a decisão de preparar um campo de estágio para o corpo discente mais próximo da prática ideal, de forma sistematizada e qualificada, sem que a assistência sofresse solução de continuidade, proporcionando à clientela prazer e satisfação com 0 atendimento de enfermagem recebido.

0 Projeto SAE recebeu o apoio do Departamento de Enfermagem da UFPI, do gestor da maternidade e da gerência de enfermagem. Esperava-se com a implantação da SAE, em uma unidade piloto, melhorar a qualidade da assistência de enfermagem dispensada às clientes internadas para tratamento clínico obstétrico no referido setor. Pretendia-se, ainda, promover a articulação ensino-serviço, através da integração interdisciplinar e multiprofissional entre docentes, discentes e outros profissionais mediante participação em eventos, reuniões cientificas semanais, capacitação em serviço, bem como estimular pesquisas na área da Saúde da Mulher.

A iniciativa agregou docentes, discentes de enfermagem da UFPI, bolsistas de enfermagem contratados pela instituição e profissionais de enfermagem da maternidade, envolvendo enfermeiras, técnicos e auxiliares de enfermagem.

Para implantar a SAE, o passo inicial envolveu a capacitação de enfermeiras da instituição, docentes e discentes da UFPI, com a colaboração de profissionais do Conselho Regional de Enfermagem do Piauí - COREN-PI. Após esta etapa, foi realizada uma oficina com os profissionais de enfermagem de nível médio para apresentação da proposta de trabalho e busca de cooperação no desenvolvimento das atividades concernentes à execução da SAE, com o foco na qualidade da assistência prestada à clientela. Além disso, foram criados grupos de trabalho, constituídos por docentes das instituições de ensino que utilizam a maternidade como campo de prática curricular e por enfermeiras assistenciais nela atuantes, para definição de impressos, organização de recursos humanos, materiais e equipamentos e elaboração de normas e rotinas.

0 Projeto foi implantado em maio de 2005. A SAE considerou inicialmente o desenvolvimento de três etapas que compõem o processo de Enfermagem: Histórico, Diagnóstico, Prescrição de Enfermagem e Evolução de Enfermagem.

Para o Histórico de Enfermagem, aplicado a todas as clientes internadas com gravidez de risco nas primeiras 24 horas, foi utilizado um formulário padronizado, preenchido por acadêmicos das disciplinas: Estágio Curricular I, II e Saúde da Mulher, da UFPI e alunos de outras faculdades de enfermagem. Constam do Histórico de Enfermagem as seguintes informações: dados de identificação; queixa atual; hábitos alimentares, atividade física e lazer; antecedentes sócio-econômicos, familiares, pessoais e gineco-obstétricos; gestação atual; exame físico por sistemas; problemas identificados.

Quanto ao Diagnóstico de Enfermagem, foi planejada a adoção da taxonomia NANDA (North American Nursing Diagnosis Association). Entretanto, esta etapa da SAE, embora considerada importante, teve a sua implantação postergada, pois alguns componentes da equipe envolvida no Projeto manifestaram não ter suficiente experiência para desenvolvêla. Para sanar a lacuna, foi programada uma agenda de estudos para dar continuidade às discussões sobre o tema, considerando que esta etapa do processo de enfermagem requer segurança e competência profissional na sua utilização.

A Prescrição de Enfermagem é realizada diariamente no turno da manhã por enfermeiros e acadêmicos de enfermagem, cujas ações prescritas foram executadas tanto por acadêmicos como por profissionais de enfermagem de nível médio da instituição, que paulatinamente aderiram ao projeto.

Para a Prescrição de Enfermagem, optou-se por formulário tipo check-list, com especificação de cuidados de enfermagem, no qual o profissional ou acadêmico assinalava todos aqueles que a cliente necessitasse, para fazer face aos problemas apresentados.

No que se refere à Evolução de Enfermagem, esta era realizada diariamente, no turno da manhã, e nos demais turnos 
eram feitas as descrições das pacientes com intercorrências e aquelas recém-admitidas no setor.

Fundamentando-se no pressuposto de que a avaliação é parte integrante de qualquer programação e deve considerar o desempenho das atividades desenvolvidas para conhecer os êxitos e os insucessos mediante estudo comparativo dos objetivos estabelecidos e os resultados alcançados ${ }^{3}$, decidiuse empreender o presente estudo.

Assim, decorridos oito meses de execução do projeto, as autoras realizaram o estudo com o objetivo de discutir aspectos relativos à implantação da SAE em uma unidade de internação de maternidade pública, segundo o ponto de vista de discentes do Curso de Enfermagem da UFPI em Estágio Curricular I e II, que participaram ativamente da iniciativa.

\section{METODOLOGIA}

A abordagem qualitativa é adequada para o presente estudo por vislumbrar a possibilidade de uma maior aproximação com a realidade dos discentes, permitindo captar o "universo de significados, motivos, aspirações, crenças, valores e atitudes", o que corresponde a um espaço mais aprofundado das ações e relações humanas ${ }^{4-22}$.

0 cenário do estudo foi uma unidade de internação de uma maternidade pública em Teresina-PI, destinada às mulheres com riscos gestacionais e puerperais, as quais permanecem internadas em média sete dias e, portanto, necessitam de maiores cuidados clínicos e de enfermagem. A maternidade, de grande porte, oferece assistência clínica e cirúrgica à mulher durante o período gravídico e puerperal, bem como ao recém-nascido normal e de risco. Possui 264 leitos obstétricos, distribuídos em nove unidades de internação, incluindo berços nos alojamentos conjunto, além de centro obstétrico e cirúrgico. É referência para todo Estado do Piauí e atende também clientela procedente de outros estados vizinhos, como o Maranhão, Pará, Tocantins e Ceará; serve de campo de estágio para alunos da área de saúde de universidades públicas, faculdades privadas e cursos de ensino médio. A produção de dados ocorreu em janeiro de 2006, e, nesta ocasião, a maternidade contava com 23 enfermeiras e 243 profissionais técnicos e auxiliares de enfermagem.

Participaram do estudo 28 acadêmicos do Curso de Enfermagem da UFPI, dos quais 24 cursavam a disciplina Estágio Curricular I e 4, o Estágio Curricular II. Estes desenvolviam atividades práticas curriculares na referida unidade, tendo participado ativamente da implantação do Projeto SAE e já haviam anteriormente cursado a disciplina Saúde da Mulher no mesmo serviço.

0 instrumento de pesquisa constou de um formulário contendo duas questões abertas: Questão I, dirigida aos alunos do Estágio Curricular I: Comente sobre sua aprendizagem no setor de tratamento clínico obstétrico da maternidade e sobre a aplicabilidade dos instrumentos da SAE adotados. Questão II, exclusiva para os alunos do Estágio Curricular II: Descreva os resultados alcançados no estágio e comente a experiência adquirida com a implantação da SAE. A aplicação do formulário ocorreu ao final do Estágio Curricular I e II e foi preenchido individualmente pelos discentes, em local reservado. Cada formulário recebeu numeração na ordem de 01 a 28 para garantir o anonimato dos sujeitos.

Foram obedecidas as normas da Resolução nº 196/96 do Conselho Nacional de Saúde. 0 projeto foi aprovado pelo Comitê de Ética em Pesquisa da UFPI, e cada participante assinou o termo de consentimento esclarecido previamente à obtenção dos dados.

A partir do relato escrito dos discentes procederam-se a leitura e releitura dos textos produzidos. Os relatos foram agrupados em categorias de acordo com a idéia principal apresentada no texto, submetidos à análise dos conteúdos segundo Bardin ${ }^{5}$ e com base no referencial teórico sobre o tema.

\section{RESULTADOS E DISCUSSÕES}

Foram evidenciadas três grandes categorias: resistência da equipe multiprofissional ao desenvolvimento do Projeto SAE, dificuldades e avanços na operacionalização da SAE e na integração multidisciplinar, e impacto na aprendizagem discente e na assistência de enfermagem.

\section{Resistência da equipe multiprofissional ao desenvolvimento do projeto SAE}

Os participantes do estudo relataram que os profissionais de saúde não estavam habituados com a presença permanente de enfermeiras e discentes no cenário escolhido para a implantação da SAE e poucos conheciam as competências técnicas e legais destas na equipe multiprofissional, o que resultou em dificuldades no desenvolvimento das atividades nos primeiros meses de implantação, tais como: falta de reconhecimento profissional e resistência da equipe de saúde às atividades realizadas, conforme ilustram os relatos a seguir:

Apesar de saber que em todo processo de mudança há resistência, a forma como os auxiliares e técnicos de enfermagem nos tratam, não valorizando, não reconhecendo o nosso trabalho... a equipe médica também...Alguns mostram-se resistentes, acham que estamos ali para brincar... Eles não vêem o nosso trabalho (Disc.2).

A maioria das dificuldades está na interdisciplinaridade, pois os outros profissionais de saúde não entendem que a enfermagem é uma profissão com autonomia e capaz de cuidar com princípios técnicos científicos (Disc.26).

Dentre as dificuldades encontradas na realização do projeto SAE, podemos citar a resistência da equipe de saúde para a concretização do mesmo. (Disc. 27).

Vale destacar que no primeiro mês de implantação do Projeto $\mathrm{SAE}$, as docentes autoras deste trabalho foram convocadas verbalmente para uma reunião com a equipe clínica da maternidade, na qual os médicos docentes, residentes e acadêmicos da área médica presentes manifestaram descontentamento pelo fato do corpo discente de enfermagem estar realizando o exame gineco-obstétrico para subsidiar 0 Histórico de Enfermagem, considerando tratar-se de atividade exclusiva do médico. Na ocasião foram esclarecidos os objetivos e metas do projeto, a competência técnica e legal da enfermeira 
na área obstétrica tanto para a realização do exame ginecoobstétrico quanto para assistir ao parto normal sem distócia, além do aval do gestor da maternidade para o desenvolvimento do Projeto.

A resistência dos profissionais de saúde em relação a SAE produziu nos alunos sentimentos de insatisfação; entretanto, não diminuiu o reconhecimento da importância do projeto para a melhoria da assistência, o que parece ser resultado de sentimento pessoal de valorização do trabalho da enfermagem, conforme se observa nos depoimentos a seguir:

Só acho que precisa melhorar ainda a satisfação dos acadêmicos como um todo, mas creio que isso virá com o reconhecimento do nosso trabalho pelos outros profissionais da casa, inclusive as enfermeiras (Disc.4).

É uma pena que nem todos os profissionais valorizem este trabalho brilhante que fazemos e que as pacientes só têm a ganhar (Disc.22).

Com o decorrer das atividades, perceberam-se avanços na integração entre acadêmicos de enfermagem e docentes envolvidos no projeto SAE com os demais profissionais de enfermagem de nível médio e equipe docente e discente da área médica. A presença permanente de enfermeiras e/ou acadêmicos de enfermagem no setor, nas 24 horas do dia, desempenhando atividades relativas ao cuidado das clientes, incorporou-se ao cotidiano da unidade e repercutiu na melhoria da assistência, planejada e sistematizada. Nesse sentido, um participante do estudo assim se manifestou:

Acho de suma importância a presença constante das enfermeiras que atuam na Maternidade para uma melhor assistência, organização, higiene do ambiente, mas sei que não vai ser fácil... (Disc. 1).

Embora o Projeto SAE tenha tido como origem a necessidade de preparar campo de prática para discentes, o envolvimento de enfermeiras da maternidade é reconhecido como vital para o gerenciamento da assistência de enfermagem, e as autoras compartilham do pensamento de que a permanência dessas profissionais garante a qualidade do processo assistencial, o que reflete a inter-relação entre atividades assistenciais e gerenciais de enfermagem, conforme enfatizam Moreira e Carvalho ${ }^{6}$.

\section{Dificuldades e avanços na integração multidisciplinar e na operacionalização da SAE}

Para a análise desta categoria é preciso mencionar a realidade vivenciada pelos acadêmicos de Enfermagem durante a disciplina Saúde da Mulher, cursada anteriormente e cujo campo de prática foi a mesma maternidade, que deixou lembranças desagradáveis ligadas à falta de acolhimento e de integração com a equipe do serviço. Assim, o retorno à instituição para cumprimento do Estágio Curricular era rejeitado pela maioria dos discentes, e a implantação do Projeto SAE permitiu abertura de novos horizontes, conforme mostram os relatos a seguir:

Pela primeira vez saí daquela maternidade com uma melhor impressão. Não vou mentir, mas a principio não sentia a menor necessidade de fazer o estágio lá, achava que já sabia o que queria saber e, no entanto, vi que aprendi muita coisa com a SAE (Disc.8).
Eu tinha uma impressão muito negativa quanto a estagiar na maternidade, mas mudei de idéia com o trabalho da SAE (Disc.24).

Como foi observado na categoria de análise anterior, havia resistência da equipe de saúde em relação à presença dos acadêmicos de enfermagem na unidade, que foi minimizada com a implantação da SAE, segundo a avaliação dos discentes.

Quanto às relações profissionais e multidisciplinares, os discentes relataram que a SAE favoreceu este processo, por sentirem-se necessários tanto para equipe de enfermagem quanto para as clientes e seus familiares, conforme se observa a seguir:

Com o projeto da SAE implantado, foi possível uma maior integração entre profissionais, familiares $e$ pacientes.... (Disc. 12).

A assistência de enfermagem na maternidade melhorou com o projeto SAE, pois aumentou a integração da equipe de enfermagem com os acadêmicos (Disc-17).

Com a SAE eu me integrei, ou tentei estar junto da equipe e das clientes. Foi muito gratificante (Disc.-1).

A importância da utilização desta metodologia científica é enfatizada por Dell'acqua e Miyadahira ${ }^{7}$ ao afirmarem que a assistência de enfermagem individualizada estreita as relações profissionais, aumentando o compromisso com o cliente, a família e a comunidade.

Entretanto, segundo o ponto de vista de alguns participantes do estudo, a integração entre os profissionais e os acadêmicos de enfermagem ainda deve melhorar, conforme mostrado a seguir:

A relação com os profissionais de algumas equipes foi ótima, já com outras foi regular (Disc.-3).

Ainda tem muito a melhorar o relacionamento entre a equipe multiprofissional, principalmente com os médicos (Disc.7).

Orelacionamento já melhorou bastante. Agora os acadêmicos de enfermagem acompanham as visitas aos leitos das pacientes internadas às 7 horas juntamente com os docentes, residentes e acadêmicos da área médica (Disc.14).

Os acadêmicos de enfermagem consideraram que a integração com a equipe multiprofissional evoluiu com o advento da SAE, melhorando significativamente as relações de trabalho no setor de implantação do projeto.

No que se refere à operacionalização do processo de enfermagem, foram descritas dificuldades relacionadas com a aplicação dos instrumentos adotados, conforme se observa nos seguintes relatos:

No início senti muita dificuldade com os instrumentos, principalmente com o Histórico. Tive dúvidas no preenchimento por ser extenso. Ele é completo, só alguns pontos a serem preenchidos me pareceram realmente duvidosos. Com o Histórico obtém-se a história completa da paciente, o seu modo de vida, antecedentes pessoais e familiares, dentre outros (Disc.5).

No que se refere à aplicabilidade dos instrumentos da $S A E$, penso que deveria haver uma diminuição nos itens do Histórico de Enfermagem. É muito cansativo para a paciente e para o aluno apesar de em grande parte tudo ser necessário (Disc. 10). 
O Histórico é muito longo. Ninguém consegue fazer em menos de meia hora. Disc. -23)

Embora o formulário do Histórico de Enfermagem tenha sido elaborado de modo objetivo, com a finalidade de reduzir o tempo destinado ao registro das atividades, ainda assim os discentes queixaram-se do tempo consumido no seu preenchimento, em média 30 minutos. Uma pesquisa realizada com enfermeiras constatou que 0 tempo médio gasto na aplicação do referido instrumento foi de 38 minutos com desvio-padrão em torno de 10 minutos ${ }^{8}$.

As autoras desta pesquisa concordam com Sperandio e Évora ${ }^{9-270}$ quando referem que os enfermeiros despendem muito tempo no preenchimento de formulários, registros de documentação da assistência de enfermagem, através de anotações manuais, e recomendam o desenvolvimento de um software para a coleta de dados e a prescrição de enfermagem que proporcione aos enfermeiros o registro informatizado de forma individualizada, eficiente e rápida.

Ainda em relação à aplicação do Histórico, um discente relatou a necessidade de espaço apropriado para realizar a entrevista (história clínica) e exame físico da cliente durante o preenchimento do formulário:

Deveria haver um local específico para o preenchimento do Histórico, pois algumas pacientes ficam constrangidas ao responder algumas questões ou serem examinadas (Disc. 13).

Vale ressaltar que no momento da entrevista recomendavase o cuidado de colocar biombo entre uma paciente e outra na enfermaria, porém esta atitude não preserva a privacidade. Benko e Castilho ${ }^{8}$ lembram a necessidade de espaço reservado para a entrevista e 0 exame físico, podendo ser uma sala com mesas e cadeiras próximas para facilitar o contato e a observação das clientes pelas enfermeiras.

Ademais, o Histórico de Enfermagem "não se caracteriza apenas por uma fase do processo de enfermagem, mas principalmente pela possibilidade de a enfermeira assumir a responsabilidade profissional por meio da utilização do saber e do fazer, exigindo ações reflexivas que demandem em compromisso e envolvimento com a assistência prestada"10-150.

Outra dificuldade relatada pelos discentes diz respeito à Prescrição de Enfermagem. Não obstante 0 embasamento teórico e prático recebido, alguns discentes mostraram-se descuidados com a prescrição de cuidados, deixando por vezes de assiná-las e executá-las, justificando a sua conduta pela escassez de recursos humanos, conforme evidenciado nas falas:

A SAE é muito bonita e resolveria muitos problemas desde que a prescrição realmente fosse executada. Acredito que ainda leva tempo até a conscientização de todos, eo aumento do numero de mão de obra também é primordial (Disc.20).

Os instrumentos da SAE são de fácil aplicação, porém, algumas vezes, a sobrecarga de trabalho no setor acaba por comprometer o desenvolvimento desta tarefa. As prescrições às vezes não são executadas e deveriam conter a assinatura de quem as fez (Disc. 13).

As dificuldades que emergiram na implementação da SAE não diferiram das encontradas em pesquisa acerca da temática, as quais mostram que a insuficiência de enfermeiras, a pouca aceitação do pessoal de enfermagem do nível médio e a complexidade das fases do processo de enfermagem levavam os acadêmicos a deixarem de executá-las quando se tornavam profissionais ${ }^{12}$. Um estudo realizado no Hospital Universitário da USP descreve a importância do quantitativo de recursos humanos no desenvolvimento da SAE, porquanto, no momento em que a assistência de enfermagem procura individualizar o cuidado dispensado ao paciente, o número de horas utilizadas para este fim varia de acordo com a complexidade do cuidado".

A SAE se constitui instrumento metodológico, ao qual se lança mão para favorecer o cuidado e organizar as condições necessárias ao desenvolvimento da prática cotidiana de enfermagem que possibilita o desenvolvimento da profissão como ciência, integrando as áreas da assistência, ensino e pesquisa.

\section{Impacto na aprendizagem discente e na assistência de enfermagem}

Esta é uma categoria que tem destaque especial pela relevância dos relatos dos sujeitos, quando relataram que a SAE ampliou o aprendizado, propiciou satisfação com o aproveitamento do Estágio Curricular l ell e melhoria na assistência de enfermagem, contrapondo com outras disciplinas cursadas anteriormente no mesmo local, conforme as manifestações a seguir:

Achei o Estágio melhor que a disciplina Saúde da Mulher por que antes a SAE ainda não estava implantada. Agora a forma de trabalho foi mais intensa... Foi muito importante, pois relembrei muitos conceitos, procedimentos técnicos e, o que é melhor, aplicando a SAE (Disc.09).

Foi de grande valia a aprendizagem neste setor, já que com a implantação da SAE passamos a prestar uma assistência com base na teoria, tendo muitas vezes que recorrer aos livros, com isso só aumentamos nossos conhecimentos (Disc.21).

É notável o crescimento intelectual de todos os acadêmicos envolvidos nesse projeto pela possibilidade de pôr em prática uma teoria tão importante para o crescimento da enfermagem como profissão (Disc.25).

Os alunos relataram que a SAE viabilizou a melhoria na qualidade da assistência prestada às clientes, em razão dos cuidados individualizados, planejados, sistematizados e baseados em evidências científicas, contribuindo para que os discentes planejassem os cuidados de enfermagem com maior segurança. Assim os discentes se expressaram:

A implantação da SAE representa um grande avanço para esta instituição que só tem a ganhar em qualidade nos serviços prestados às pacientes internadas (Disc.3).

A SAE permite assistência de enfermagem diferenciada, individualizada, gera satisfação do profissional e da cliente (Disc. 12).

Com a implantação da SAE na maternidade, pode-se perceber uma melhoria na qualidade da assistência prestada às pacientes, uma assistência continuada, baseada nas necessidades de cada uma (Disc.25). 
Com poucos meses do início deste projeto já é visível a melhoria na assistência de pacientes que necessitam de tratamento clínico na gestação e no puerpério nesta maternidade (E-26).

A implantação da SAE nos serviços de saúde dá visibilidade ao trabalho dos profissionais de enfermagem envolvidos e favorece 0 reconhecimento, pelos demais membros da equipe de saúde, familiares e clientela assistida, do trabalho desenvolvido.

No relato a seguir observam-se a percepção do discente acerca da importância da SAE e a necessidade de expansão para outros setores da maternidade e demais instituições:

Implementar a SAE em um serviço que presta cuidados à saúde da clientela deve ser prioridade, pois os cuidados planejados pelo enfermeiro resultam em melhoria da assistência, comprometimento da equipe de enfermagem e clientela satisfeita com o cuidado. A SAE deve ser colocada em prática, não só na maternidade, mas em todos os serviços de saúde (Disc.28).

A repercussão da experiência com a SAE na unidade piloto favoreceu a adesão de alunos interessados em realizar o Estágio Curricular II na maternidade, bem como o interesse das enfermeiras em ampliar a SAE para outros setores da instituição, especialmente a UTI. Entretanto, Amorim e Carvalho ${ }^{13}$, em estudo realizado nesta mesma maternidade, afirmaram que as enfermeiras desconhecem o processo de enfermagem, em virtude da pouca vivência prática com a SAE durante a vida acadêmica, e apontam como dificuldades para a adoção desta metodologia assistencial: a falta de recursos humanos e de normalização do serviço, que as induz a priorizar tarefas burocráticas. Para reverter a situação existente, sugerem a expansão e capacitação da força de trabalho de enfermagem.

\section{CONSIDERAÇÕES FINAIS}

Esta pesquisa foi produto de reflexão das autoras sobre a qualidade da formação acadêmica de enfermagem na UFPI em face da ausência de um campo de prática que desenvolva a sistematização da assistência de enfermagem em Teresina. Com a proposta de implantação do projeto SAE em uma unidade de internação da maternidade pública, buscou-se adotar etapas do processo de enfermagem como instrumento metodológico para favorecer o cuidado, organizar as condições necessárias para o desenvolvimento da prática cotidiana voltada para 0 desenvolvimento da profissão como ciência, integrando as áreas da assistência, ensino e pesquisa.

\section{Referências}

1. Avelino FPSD. 0 ensino da sistematização da assistência na visão crítica do egresso da graduação em Enfermagem. [dissertação de mestrado]. Teresina (PI): Centro de Ciências da Educação /UFPI; 2003.

2. Conselho Federal de Enfermagem- COFEn. Resolução n 272 de 27 de agosto de 2002. Dispõe sobre a Sistematização da Assistência de Enfermagem- SAE nas instituições de saúde brasileiras. Brasilia(DF); 2002.Arts 1-3.
0 estudo evidenciou que, a despeito da resistência inicial dos profissionais de saúde com o trabalho desenvolvido no Projeto $\mathrm{SAE}$, o resultado foi relevante para o ensino e para a construção da identidade profissional.

Com a implantação da Sistematização da Assistência, os discentes relataram 0 alcance dos objetivos programados, refletido na melhoria do aprendizado e da assistência de enfermagem, favorecida pela integração com a equipe multiprofissional, em especial com os técnicos e auxiliares de enfermagem, e percepção da satisfação dos familiares e da clientela com os cuidados recebidos.

No que se refere à operacionalização da SAE, foram destacadas dificuldades relativas ao tempo consumido com 0 preenchimento dos formulários e manuseio de alguns instrumentos. Em relação ao Histórico de Enfermagem, evidenciou-se a necessidade de fortalecer os conhecimentos teóricos e práticos dos discentes, principalmente no que se refere à realização do exame físico, levantamento de problemas/diagnósticos e condutas de enfermagem.

Referente à Prescrição de Enfermagem, há que se enfatizar a necessidade de maior compromisso com a identificação do executor dos cuidados prescritos, prática relevante na administração da assistência de enfermagem e na avaliação da qualidade assistencial. Evidenciou-se também a necessidade de utilizar 0 Diagnóstico de Enfermagem, como etapa integrante da SAE.

A implantação do Projeto SAE contribuiu para o fortalecimento da autonomia e satisfação dos alunos, evidenciada na mudança de atitude dos discentes quanto à valorização da metodologia no seu cotidiano, bem como com a redução do sentimento de rejeição da maternidade como campo de prática.

Como sugestão, os discentes referiram a expansão da SAE para outros setores da maternidade, embora reconheçam que a escassez de recursos humanos qualificados é um obstáculo a ser superado.

As autoras esperam que a implantação da SAE efetivamente contribua para que os discentes sejam agentes multiplicadores da experiência nos serviços de saúde nos quais irão atuar profissionalmente.

Os resultados alcançados com o projeto SAE repercutiram no ensino e favoreceram a inserção da disciplina Metodologia da Assistência de Enfermagem no novo currículo do Curso de Enfermagem - Bacharelado da UFPI, implantado em 2007. Tal iniciativa necessita do desenvolvimento de parcerias com os hospitais de ensino no sentido de viabilizar a SAE e oferecer aos discentes experiências de aprendizagem que focalizem 0 cuidado sistematizado e, aos usuários, possibilita assistência de enfermagem de melhor qualidade.

3. Rocha CMV. Avaliação de programas de saúde. Rev Assoc Saúde Pública, Piauí, 1999 jan/jun; 2(1): 71-77.

4. Minayo MCS. 0 desafio do conhecimento: pesquisa qualitativa em saúde. $6^{\text {a }}$ ed. São Paulo (SP), Rio de Janeiro (RJ): Hucitec; 2006.

5. Bardin L. Análise de conteúdo. São Paulo (SP): Ed Loyola 70; 1977.

6. Moreira MC, Carvalho V. Relações de ajuda: reflexões sobre a aplicabilidade no processo assistencial de enfermagem. Esc Anna Nery Rev Enferm 2004 dez; 8(3): 354-60. 
7. Dell'acqua MCQ, Miyadahira AMK. Ensino do processo de enfermagem nas escolas de graduação em enfermagem do Estado de São Paulo. Rev Latino-am Enfermagem 2002 abr; 10(2): 185 -91.

8. Benko MA, Castilho V. Operacionalização de um sistema de assistência de enfermagem. In: Campedelli MC, organizadora. Processo de enfermagem na prática. São Paulo (SP): Ática; 1989.

9. Sperandio DJ, Évora YDM. Sistematização da assistência de enfermagem: proposta de um software - protótipo. Esc Anna Nery Rev Enferm 2003 ago; 7(3): 269-82.

10. Gutierrez BAO, Lopes AL, Cruz DALM, Souza TT. Histórico de enfermagem. In: Cianciarullo TI, Gualda DMR, Melleiro MM, Anabuki MH. Sistema de assistência de enfermagem: evolução e tendências. São Paulo (SP): Ícone; 1999.

11. Garcia TR, Nobrega MML. Sistematização da assistência de enfermagem: reflexões sobre o processo. Anais do $52^{\circ}$ Congresso Brasileiro de Enfermagem; 2000 out 21-26; Recife-Olinda (PE), Brasil. Recife (PE): ABEn; 2000. p 231-34.

12. Campedelli MC, Taquiko C, Sancinetti TR, Benko MA. Cálculo de pessoal de enfermagem: competência da enfermeira. Rev Esc Enferm USP $1987 \mathrm{abr} ; 21(1):$ 3-15.
13. Amorim FCM, Carvalho MGPV. Opinião das enfermeiras sobre aplicabilidade do processo de enfermagem em uma maternidade pública de Teresina - PI. [monografia de especialização em Enfermagem Obstétrica]. Teresina (PI): Centro de Ciências da Saúde/ UFPI; 2004.

\section{Sobre as Autoras}

\section{Lucimar Ramos Ribeiro Gonçalves}

Enfermeira Obstetra, Mestre em Enfermagem. Professora Adjunta IV do Departamento de Enfermagem da Universidade Federal do Piauí.

\section{Inez Sampaio Nery}

Doutora em Enfermagem. Professora Adjunta IV do Departamento de Enfermagem da Universidade Federal do Piauí.

\section{Lídya Tolstenko Nogueira}

Doutora em Enfermagem. Professora Adjunta IV do Departamento de Enfermagem da Universidade Federal do Piauí.

\section{Elisiane Gomes Bonfim}

Professora Substituta da Universidade Federal do Piauí, Mestranda em Enfermagem. 